\title{
Georges David
}

Né en Lorraine en 1923, le Professeur Georges David a fait ses études de médecine à Paris où il s'était exilé avec sa famille afin d'éviter d'être enrôlé de force dans l'armée allemande pendant la $2^{\mathrm{e}}$ guerre mondiale. Il avait prévu de devenir médecin généraliste en Normandie avec sa jeune épouse qui était sage-femme mais son destin fut contrarié. En effet, en attendant de s'installer, Georges David participait au développement de l'exsanguinotransfusion des nouveau-nés pour lutter contre les incompatibilités fœeto-maternelles aux côtés de Sylvain Buhot. Quand ce dernier décéda accidentellement, il fut demandé à Georges David de le remplacer et c'est ainsi qu'il devint un expert reconnu de la transfusion sanguine de l'hôpital SaintAntoine à Paris. Curieux et passionné, il menait des recherches dans le domaine et c'est ainsi qu'il trouva le moyen de traiter l'anasarque foto-placentaire qui était souvent fatale pour les enfants qui en étaient atteints.

Plus tard, il a rejoint l'équipe du Pr Petit à Saint-Vincent de Paul, où il pratiqua l'anesthésie lors d'interventions chirurgicales particulièrement lourdes chez l'enfant et où il participa au développement de la réanimation néonatale qui en était à ses débuts. Devenu assistant au Laboratoire d'Embryologie de la Faculté de Médecine, il fut nommé professeur agrégé en 1965. Quelques années plus tard, quand il devint le représentant de la discipline au sein du CHU du Kremlin Bicêtre et qu'il lui fallait trouver une insertion hospitalière, il eut l'idée de créer un laboratoire d'étude de l'infertilité masculine. Ce projet, tout à fait original à l'époque, avait été notamment influencé par les conseils de Maurice Lacomme, chef du service de gynécologie-obstétrique à l'hôpital Saint-Antoine où il avait débuté sa carrière professionnelle.

Pour identifier les causes de l'infertilité, Georges David et son équipe ont mis au point des techniques biologiques novatrices basées essentiellement sur l'analyse du sperme, permettant d'identifier la cause masculine de la stérilité et évitant de faire porter le poids de l'infertilité à la seule femme, comme c'était souvent le cas à l'époque. Cependant, dans la plupart des cas, il n'y avait pas de traitement efficace pour restaurer une spermatogenèse fonctionnelle et la fertilité. Les couples stériles étaient souvent amenés à recourir à l'insémination artificielle avec sperme de donneur (IAD) dans des conditions aussi douteuses qu'incertaines, confronté à leur détresse, Georges David a imaginé de créer dès 1973 un
CECOS (Centre d'Étude et de la Conservation des Oeufs et du Sperme) ayant pour but de prendre en charge ces couples et de traiter cette difficile question avec des moyens médicaux exigeants et selon des principes éthiques rigoureux. Les donneurs étaient bénévoles et étaient soumis à des tests afin d'éviter au mieux la transmission de pathologies. Leur sperme, systématiquement congelé, était utilisé de manière anonyme. Maîtrisant la congélation du sperme, le CECOS a très vite proposé d'utiliser aussi cette technique pour préserver la fertilité quand elle était menacée par une intervention médicale (traitements anticancéreux, vasectomie...). L'expérience du CECOS de Bicêtre a rapidement connu une notoriété internationale et a bénéficié à de nombreux autres CHU en France. C'est ainsi que s'est constitué un réseau national unique au monde qui existe encore aujourd'hui (www.cecos.org).

Les engagements de Georges David ont été marqués tout au long de sa carrière par l'ouverture, l'innovation et la rigueur : ouverture vers des champs d'activité médicale peu explorés auparavant, innovation par le développement de nouvelles technologies biologiques, rigueur dans leur utilisation dont il souhaitait qu'elle soit évaluée, ce qui fut fait grâce à la collaboration fructueuse menée avec Daniel Schwartz, «père» de la statistique médicale et de l'épidémiologie française.

Menant ses activités avec beaucoup d'humanité et dans le plus grand respect des patients comme de ses collègues, Georges David a marqué de son empreinte l'évolution de la biologie et de la médecine de la fin du $\mathrm{xx}^{\mathrm{e}}$ siècle. Parmi les traces qu'il a laissées, on peut citer la création d'une discipline, la biologie de la reproduction qui a été aussi l'ouverture de nouvelles perspectives pour la biologie médicale, celles d'une biologie qui ne soit plus uniquement diagnostique mais qui soit aussi à visée thérapeutique: la biologie interventionnelle. On ne peut ignorer non plus que les règles déontologiques qui avaient été édictées par les CECOS ont inspiré en grande partie les principes inscrits dans la loi relative à la bioéthique en 1994 et sont encore en vigueur aujourd'hui.

NB : un film intitulé «Georges David, un médecin du $\mathrm{xx}^{\mathrm{e}}$ siècle » a été réalisé il y a quelques années. Il s'agit d'un entretien où il parle de son parcours professionnel. Il est libre d'accès sur Youtube: https://www.youtube.com/ watch? $\mathrm{v}=$ jzzYRRa_bi8. 